\title{
Low-frequency fluctuations in vertical-cavity surface-emitting semiconductor lasers with optical feedback
}

\author{
C. Masoller ${ }^{1,2}$ and N. B. Abraham ${ }^{2,3}$ \\ ${ }^{1}$ Instituto de Física, Facultad de Ciencias, Universidad de la República, Igua 4225, Montevideo 11400, Uruguay \\ ${ }^{2}$ Department of Physics, Bryn Mawr College, 101 North Merion Avenue, Bryn Mawr, Pennsylvania 19010-2899 \\ ${ }^{3}$ DePauw University, Greencastle, Indiana 46135-0037
}

(Received 28 September 1998)

\begin{abstract}
We study the dynamics of a vertical-cavity surface-emitting laser operating near threshold and with isotropic optical feedback, using a model developed by San Miguel, Feng, and Moloney [Phys. Rev. A 52, 1728 (1995)]. The model couples the polarization state of the electric field to the semiconductor medium by including the magnetic sublevels of the conduction and valence bands in the quantum wells. The laser dynamics depend significantly on the value of the relaxation rate, $\gamma_{s}$, of the material magnetization. For low relaxation rates the time-averaged intensity abruptly drops to zero and then recovers, a phenomenon revealed to be a sequence of picosecond pulses. The dropouts are similar to those occurring in conventional semiconductor lasers, but underlying the dropouts there is an antiphase competition between the time-averaged orthogonal linearly polarized components of the electric field. For large values of the relaxation rate, the dropouts tend to disappear and the time-averaged intensity is nearly constant. [S1050-2947(99)09603-1]
\end{abstract}

PACS number(s): 42.55.Px, 05.45.-a, 42.65.Sf

\section{INTRODUCTION}

A vertical-cavity surface-emitting laser (VCSEL) is a relatively new type of semiconductor laser that has a different geometry from that of a conventional (edge-emitting) laser diode. A VCSEL consists of a semiconductor laser gain medium sandwiched between two highly reflective mirrors, thus the laser output occurs normal to the wafer. VCSEL's have several advantages for applications. Because of their short cavity length (few $\mu \mathrm{m}$ ), VCSEL's emit in a single longitudinal mode. They have low threshold currents and can be modulated at high speeds. They are compact, thus it is easy to form laser arrays.

An important drawback to applications of VCSEL's is the polarization instability. The output of a VCSEL is usually linearly polarized along one of two orthogonal directions associated with crystalline or stress orientations. When the VCSEL begins to lase, one linear polarization dominates and when the injection current is increased about $20-30 \%$ above the lasing threshold, in many devices the emission switches to the other polarization state (polarization switching) [1]. These two linearly polarized states have different optical frequencies, split (from 2 to $20 \mathrm{GHz}$ ), by the material birefringence. Sometimes there is simultaneous emission on both of the orthogonal linearly polarized states with different emission frequencies (polarization coexistence) [2], and there are a few reports of emission on both orthogonal linearly polarized states with the same emission frequency (elliptically polarized light) [3]. If the injection current is increased further, higher-order transverse modes emerge, usually with a polarization state that is orthogonal to the polarization of the fundamental mode [4].

An explanation for the polarization switching phenomenon, proposed by Choquette and co-workers [2,5], relies on variations in the gain profile and in the optical frequencies of the orthogonal linearly polarized states, induced by tempera- ture changes as the injection current increases.

An alternative explanation of the polarization switching is based on the San Miguel, Feng, and Moloney (SFM) model [6]. In conventional edge-emitting semiconductor lasers the anisotropies, geometry, and waveguiding effects of the cavity lead to emission of linearly polarized light with the polarization locked onto a fixed axis. However, the polarization state of the light of a laser is linked, not only to cavity anisotropies, but also to the field-material physics (to the angular momentum of the quantum states involved in the transitions for emission and absorption [7]). The SFM model takes into account the spin sublevels of the conduction and valence band and distinguishes the amplitudes of the orthogonal circular polarizations of the light that are associated with transitions between different spin sublevels. In the absence of anisotropies, the stationary solutions of the SFM model are linearly polarized states with arbitrary polarization direction. In the presence of anisotropies the direction of the linear polarization is no longer arbitrary, and the stationary solutions are two orthogonal linearly polarized states. The relative strength of the gain for the two states and the birefringence and saturable dispersion of the material determine the stability of these states. A linear stability analysis explains the polarization switching for increasing injection current $[8,9]$.

Physical insight into the nature of the polarization switching was recently put forward by van der Lem and Lenstra [10] and van Exter et al. [11], who showed analytically (by adiabatically eliminating the spin dynamics) that the presence of a strong lasing state with a certain polarization leads to a broadening and redshift of the weak orthogonal nonlasing state.

The spin dynamics is especially relevant when a VCSEL is in an externally applied magnetic field. In that case the SFM model predicts emission in an elliptically polarized state $[12,13]$, which was verified experimentally [3]. The switching between orthogonal linearly polarized states then 
becomes switching between elliptically polarized states [14].

This polarization instability enhances the intensity noise [15] and degrades polarization sensitive applications such as magneto-optic disk recording. However, since the polarization switching occurs very quickly and requires only small variations of the injection current, the polarization state can be a good property to choose for optical logic gates. Methods to control the polarization state of a VCSEL include breaking the transverse devise symmetry (by introducing anisotropic gain [16] or loss [17], or by using an asymmetric cavity geometry [18]), optical injection [19], current modulation about the polarization switching point [20], and external optical feedback [21-24].

Optical feedback is an especially attractive way to achieve polarization switching. By coupling a VCSEL to an external microcavity (with an external cavity length of the order of microns), a single transverse mode operation can be maintained even at high injection currents [25]. Slight movements of an external mirror can tune a VCSEL in wavelength [26]. However, in a conventional linearly polarized single-mode semiconductor laser it is well known that uncontrolled optical feedback degrades the performance by exciting additional external cavity modes (i.e., the stationary solutions of the compound system formed by the laser cavity and the external cavity), which may lead to a chaotic state termed "coherence collapse"' (see, e.g., [27,28] and references therein). It could be expected that the high facet reflectivity of a VCSEL (of the order of 99\%) might mitigate the effects of backreflections. However, the extremely short cavity length of a VCSEL (of the order of microns) cancels out the effect of highly reflective mirrors, and VCSEL's usually display a feedback sensitivity comparable to that of conventional semiconductor lasers [29-34]. The threshold current is reduced by external feedback, and the five characteristic regimes of feedback that occur in edge-emitting lasers [35] also occur in VCSEL's.

A well-known feedback-induced instability is the lowfrequency fluctuations (LFF's), which occur for moderate feedback when the laser is operated near the lasing threshold. These consist of sudden power dropouts followed by gradual, stepwise recoveries [36-38]. Fischer and coworkers [39] observed with a streak camera that the intensity dropouts are actually a slow modulation of a series of fast, picosecond pulses. The model commonly used to interpret the experimental observations is the Lang-Kobayashi (LK) model [40] for a single-mode semiconductor laser. Numerical simulations of the LK model show that the time-averaged laser intensity exhibits dropouts similar to those observed experimentally, and that underlying these dropouts there is a fast pulsing behavior [39]. However, recent experimental statistical analysis of the intensity fluctuations during the dropouts [41] and of the statistical distribution of the time intervals between power dropouts [42] shows discrepancies with the predictions of the single-mode model. Moreover, the experimental observations of Huyet et al. [43] indicate that the emergence of low-frequency fluctuations is associated with the excitation of several longitudinal modes of the solitary laser. In this paper we investigate the occurrence of LFF's in VCSEL's.

Computational studies of VCSEL's with optical feedback have often used models that do not consider the vectorial nature of VCSEL's, i.e., they consider several transverse modes but do not take into account the fact that two orthogonal linearly polarized states exist for each tranverse mode [44-46]. These models are those used for conventional, polarization-stabilized semiconductor lasers, though with different parameter values. However, when a VCSEL with optical feedback is operated near the lasing threshold (where the polarization switching often takes place), one would expect that the polarization instability would influence the dynamics significantly. To take into account the polarization degree of freedom, we simulate the dynamics based on the SFM model, which considers a single transverse mode with two orthogonal linearly polarized states, $E_{x}, E_{y}$, coupled through two carrier populations (the total carrier population, $N$, and the population difference, $n$, between the spin sublevels of the conduction and valence bands). The SMF model has been successfully used to study the dynamics of a VCSEL under polarized feedback (into one of the two linearly polarized states $[47,48]$ ) and under polarization-changing feedback (with a quarter-wave plate placed in the external cavity [49]). With polarized feedback the SMF model predicts the observed polarization switchings [21] when the injection current or the feedback phase varies. With polarization-changing feedback, the intensity waveforms predicted by the model agree qualitatively well with the experimental measurements [22].

Another proposed model for polarization dynamics in VCSEL's describes the interaction of the two linearly polarized states of the fundamental mode through a single carrier density and nonlinear gain terms. The predictions of this model for polarized feedback [47] and for polarizationchanging feedback [24] agree with the experimental observations and with the predictions of the SFM model. Here we compare the predictions of these two models in the LFF's regime.

This paper is organized as follows. Section II presents a brief description of the SMF model with isotropic optical feedback. Section III contains the results of the numerical simulations. We study the time evolution of the total intensity, of the intensity of the orthogonal linearly polarized components, and of the intensity of the circularly polarized components. The dynamics of the polarization state of the light is revealed with the Poincare-sphere representation [50]. Section IV contains a summary and the conclusions.

\section{THEORETICAL MODEL}

The rate equations for a VCSEL with external isotropic feedback operating in the fundamental transverse mode are [6]

$$
\begin{aligned}
\dot{E}_{x}= & k(1+i \alpha)\left[(N-1) E_{x}+i n E_{y}\right] \\
& -\left(\gamma_{a}+i \gamma_{p}\right) E_{x}+\gamma E_{x}(t-\tau) e^{-i \omega_{o} \tau}, \\
\dot{E}_{y}= & k(1+i \alpha)\left[(N-1) E_{y}-i n E_{x}\right] \\
& +\left(\gamma_{a}+i \gamma_{p}\right) E_{y}+\gamma E_{y}(t-\tau) e^{-i \omega_{o} \tau}, \\
\dot{N}= & -\gamma_{N}\left[N-\mu+N\left(\left|E_{x}\right|^{2}+\left|E_{y}\right|^{2}\right)\right. \\
& \left.+i n\left(E_{y} E_{x}^{*}-E_{x} E_{y}^{*}\right)\right],
\end{aligned}
$$




$$
\dot{n}=-\gamma_{s} n-\gamma_{N}\left[n\left(\left|E_{x}\right|^{2}+\left|E_{y}\right|^{2}\right)+i N\left(E_{y} E_{x}^{*}-E_{x} E_{y}^{*}\right)\right],
$$

where $E_{x}, E_{y}$ are the slowly varying amplitudes of the orthogonal linearly polarized components of the electric field, $N$ is the total population difference between conduction and valence bands, and $n$ is the population difference between the carrier densities with positive and negative spin values. $k$ is the field decay rate $(1 / 2 k$ is sometimes called the photon lifetime), $\gamma_{N}$ is the decay rate of the total carrier population, and $\gamma_{s}$ is the decay rate which accounts for the mixing of the populations with different spins. $\alpha$ is the linewidth enhancement factor, which indicates the dependence of the index of refraction on the carrier number [51]. $\mu$ is the normalized injection current (which takes the value of 1 at the solitary laser threshold) and $\omega_{o}$ is the optical frequency of the solitary laser at the lasing threshold in the absence of linear anisotropies.

The model incorporates the effects of isotropic optical feedback through a delayed term in Eqs. (1) and (2). We follow the Lang-Kobayashi approach [40] and consider a single reflection in the external cavity. $\gamma$ is the feedback intensity and $\tau=2 L / c$ is the round-trip time in the extended cavity of length $L$.

Usually the two orthogonal linearly polarized steady-state solutions have different optical frequencies, which are associated with the birefringence of the medium, and different gain-to-loss ratios. These effects are modeled by the parameters $\gamma_{a}$ and $\gamma_{p}$. Positive $\gamma_{a}$ gives the $y$-polarized state a lower threshold, and $\gamma_{p}$ leads to a frequency difference of $\gamma_{p} / \pi$ between the $x$ - and $y$-polarized states in the absence of gain anisotropies and external feedback (when $\gamma_{p}$ is positive the $x$-polarized state has the lower frequency).

Taking $n=0, E_{x}=E_{y}=E / 2$, and $\gamma_{a}=\gamma_{p}=0$, Eqs. (1)-(4) reduce to the familiar equations for a single-mode polarization-stabilized semiconductor laser with optical feedback (where $N$ is the difference between the actual carrier density and the carrier density at transparency).

The parameter $\gamma_{s}$ models the population equilibration of the magnetic sublevels due to a variety of complicated microscopic processes termed spin-flip relaxation processes. Experimental measurements of spin relaxation times in quantum wells [52,53] yield values for $\gamma_{s}$ of the order of $10 \mathrm{~ns}^{-1}$. However, such values apply to VCSEL's operating at low temperature; at room temperature, $\gamma_{s}$ is expected to be of the order of $100 \mathrm{~ns}^{-1}$ [13].

To study the influence of spin relaxation, we consider two limiting cases. First, the mathematical limit of very large $\gamma_{s}$ (very fast mixing of populations with different spins), which is equivalent to setting $n$ equal to zero in Eqs. (1)-(4). One then obtains the following equations in which the two modal amplitudes $E_{x}, E_{y}$ are coupled to a single carrier population $N$,

$$
\begin{gathered}
\dot{E}_{x}=k(1+i \alpha)(N-1) E_{x}-\left(\gamma_{a}+i \gamma_{p}\right) E_{x}+\gamma E_{x}(t-\tau) e^{-i \omega_{o} \tau}, \\
\dot{E}_{y}=k(1+i \alpha)(N-1) E_{y}+\left(\gamma_{a}+i \gamma_{p}\right) E_{y}+\gamma E_{y}(t-\tau) e^{-i \omega_{o} \tau}, \\
\dot{N}=-\gamma_{N}\left[N-\mu+N\left(\left|E_{x}\right|^{2}+\left|E_{y}\right|^{2}\right)\right] .
\end{gathered}
$$

Second, when $\gamma_{s}$ takes its minimum value (given by the radiative lifetime of carriers, $\gamma_{s}=\gamma_{N}$ ) one then obtains two modal amplitudes $E_{+}, E_{-}$coupled to two carrier populations $N_{+}, N_{-}$,

$$
\begin{aligned}
\dot{E}_{ \pm}= & k(1+i \alpha)\left(N_{ \pm}-1\right) E_{ \pm}-\gamma_{a} E_{\mp}-i \gamma_{p} E_{\mp} \\
& +\gamma E_{ \pm}(t-\tau) e^{-i \omega_{o} \tau} \\
& \dot{N}_{ \pm}=-\gamma_{N}\left[N_{ \pm}-\mu+2 N_{ \pm}\left|E_{ \pm}\right|^{2}\right],
\end{aligned}
$$

where $E_{ \pm}=E_{x} \pm i E_{y}$ are the slowly varying amplitudes of the left and right circularly polarized components of the electric field, and $N_{ \pm}=N \pm n$.

For each linearly polarized state of the solitary laser, optical feedback creates a series of new stationary solutions, which are analogous to the so-called external cavity modes of conventional lasers with optical feedback $[27,28]$. They are of the form

$$
E_{ \pm}=E_{s}^{i} \exp \left[i\left(\omega_{s}^{i}-\omega_{o}\right) t \pm \varphi^{i}\right], \quad N=N_{s}^{i}, \quad n=0,
$$

where $E_{s}^{i}, \varphi^{i}$, and $N_{s}^{i}$ satisfy for the $x$-polarized state,

$$
\varphi^{i}=0, \quad N_{s}^{i}=1+\frac{\gamma_{a}-\gamma \cos \omega_{s}^{i} \tau}{k}, \quad E_{s}^{i}=\left(\frac{\mu-N_{s}^{i}}{N_{s}^{i}}\right)^{1 / 2}
$$

and $\omega_{s}^{i}$ is a solution of

$$
\omega_{s}^{i}-\omega_{o}=\alpha \gamma_{a}-\gamma_{p}-\gamma\left(\alpha \cos \omega_{s}^{i} \tau+\sin \omega_{s}^{i} \tau\right),
$$

and for the $y$-polarized state,

$$
\begin{gathered}
\varphi^{i}=\pi / 2, \quad N_{s}^{i}=1-\frac{\gamma_{a}+\gamma \cos \omega_{s}^{i} \tau}{k}, \quad E_{s}^{i}=\left(\frac{\mu-N_{s}^{i}}{N_{s}^{i}}\right)^{1 / 2}, \\
\omega_{s}^{i}-\omega_{o}=-\alpha \gamma_{a}+\gamma_{p}-\gamma\left(\alpha \cos \omega_{s}^{i} \tau+\sin \omega_{s}^{i} \tau\right)
\end{gathered}
$$

Stationary solutions that correspond to elliptically polarized states also exist. They are of the form

$$
\begin{gathered}
E_{x}=E_{x} e^{i\left(\omega_{s}-\omega_{o}\right) t+i \varphi_{x}}, \quad E_{y}=E_{y} e^{i\left(\omega_{s}-\omega_{o}\right) t+i \varphi_{y}}, \\
N=N_{s}, \quad n=n_{s},
\end{gathered}
$$

where $E_{x}, E_{y}, N_{s}, n_{s}, \omega_{s}$, and $\Delta \varphi=\varphi_{x}-\varphi_{y}$ verify

$$
\begin{aligned}
& k\left[\left(N_{s}-1\right) E_{x}+n_{s} E_{y}(\sin \Delta \varphi-\alpha \cos \Delta \varphi)\right]-\gamma_{a} E_{x} \\
& \quad+\gamma E_{x} \cos \omega_{s} \tau=0, \\
& k\left[\alpha\left(N_{s}-1\right) E_{x}+n_{s} E_{y}(\cos \Delta \varphi+\alpha \sin \Delta \varphi)\right] \\
& \quad-\gamma_{p} E_{x}-\gamma E_{x} \sin \omega_{s} \tau \\
& \quad=E_{x}\left(\omega_{s}-\omega_{o}\right),
\end{aligned}
$$

$$
\begin{aligned}
& k\left[\left(N_{s}-1\right) E_{y}+n_{s} E_{x}(\sin \Delta \varphi+\alpha \cos \Delta \varphi)\right]+\gamma_{a} E_{y} \\
& \quad+\gamma E_{y} \cos \omega_{s} \tau=0,
\end{aligned}
$$




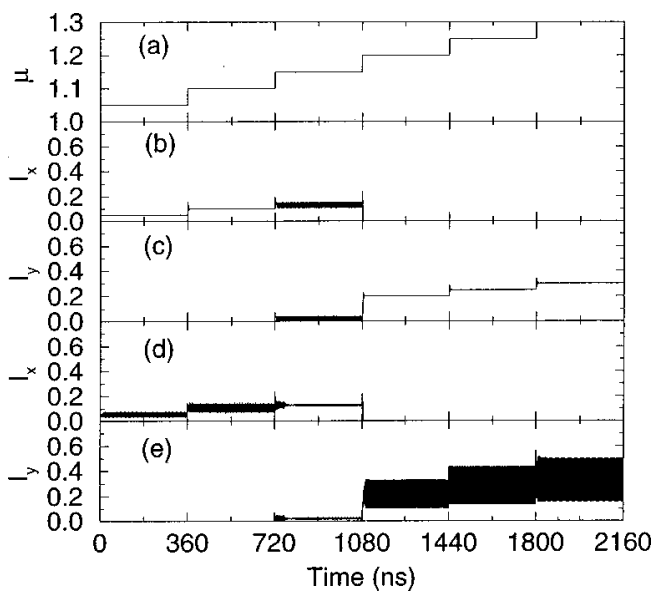

FIG. 1. Polarization switching for increasing injection current. The parameters are $\gamma_{a}=0.1 \mathrm{~ns}^{-1}, \gamma_{p}=2 \mathrm{~ns}^{-1}$, and $\gamma_{s}=50 \mathrm{~ns}^{-1}$. (a) Time-dependent normalized injection current $\mu$. (b),(c) Intensities (in arbitrary units) of the $x$ - and $y$-polarized components, respectively, without feedback. (d), (e) Intensities (in arbitrary units) of the $x$ - and $y$-polarized components, respectively, with a feedback level $\gamma=0.5 \mathrm{GHz}$.

$$
\begin{aligned}
& k\left[\alpha\left(N_{s}-1\right) E_{y}-n_{s} E_{x}(\cos \Delta \varphi-\alpha \sin \Delta \varphi)\right] \\
& +\gamma_{p} E_{y}-\gamma E_{y} \sin \omega_{s} \tau=E_{y}\left(\omega_{s}-\omega_{o}\right), \\
& -\mu+N_{s}\left(1+E_{x}^{2}+E_{y}^{2}\right)+2 n_{s} E_{x} E_{y} \sin \Delta \varphi=0, \\
& \quad n_{s}\left(\frac{\gamma_{s}}{\gamma_{N}}+E_{x}^{2}+E_{y}^{2}\right)+2 N_{s} E_{x} E_{y} \sin \Delta \varphi=0 .
\end{aligned}
$$

The symmetry of these equations implies that two elliptically polarized states exist with the same optical frequency $\omega_{s}$. They have opposite values of $n_{s}$, and their values of $\Delta \varphi$ differ by $\pi$.

\section{NUMERICAL RESULTS}

We solved Eqs. (1)-(4) with weak stochastic noise perturbations added to each of the variables (of strength $\beta$ $=10^{-4} \mathrm{~ns}^{-1}$ ) and an integration step of $0.5 \mathrm{ps}$ (as discussed in Ref. [47], in many cases without noise terms no polarization switching is observed). The initial conditions are taken in the $x$ - or $y$-polarized states of the solitary laser, and the parameters chosen in our simulation are the same as in Ref. [9]: $\quad \alpha=3, k=300 \mathrm{~ns}^{-1}, \quad \gamma_{N}=1 \mathrm{~ns}^{-1}, \tau=3 \mathrm{~ns}$, and $\omega_{o} \tau$ $=6 \mathrm{rad}$. The parameters $\gamma_{a}, \gamma_{p}, \gamma_{s}, \gamma$, and $\mu$ are free parameters, varied to study the different dynamical regimes. Conditions of monostability, bistability, and dynamical instability of the laser without feedback were studied in detail in [9]. It was shown there that the saturable dispersion (represented by the $\alpha$ factor) and birefringence determine the stability of the linearly polarized states when they have approximately the same gain-to-loss ratio. Here we study how optical feedback modifies these conditions.

\section{A. Dynamics for weak optical feedback}

Figures 1 and 2 show the effect of weak optical feedback on the polarization switching that occurs for $\gamma_{a}=0.1 \mathrm{~ns}^{-1}$ as the injection current is increased. (The injection current was

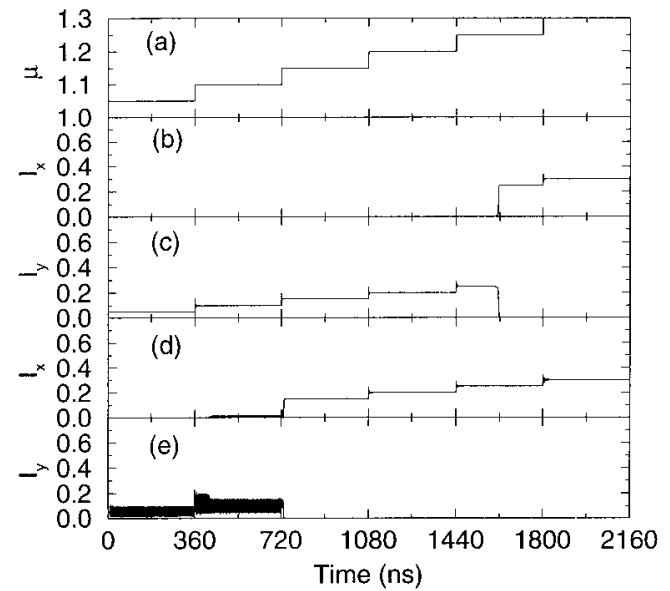

FIG. 2. Polarization switching for increasing injection current with $\gamma_{p}=10 \mathrm{~ns}^{-1}$. All other features are the same as in Fig. 1.

increased in steps; each new value was held constant for 120 round trips in the external cavity, 360 ns to let transients die away.) The intensities of the linearly and circularly polarized components of the electric field are defined as $I_{x, y}=\left|E_{x, y}\right|^{2}$, $I_{ \pm}=\left|E_{ \pm}\right|^{2}$.

Under weak feedback levels, the polarization switchings that occur for increasing injection current are similar to those that occur for the VCSEL without feedback, but the values of the injection current at which the polarization switchings occur are slightly different from those for the solitary laser. Figure 1 shows polarization switching from the $x$-polarized state to the $y$-polarized state. In the absence of feedback [see Figs. 1(b) and 1(c)] for increasing injection current the $x$-polarized state loses stability and a state of mixed polarization (with a periodic modulation of the intensities of the linearly polarized components) appears [9]. For larger injection current the system switches to the $y$-polarized state. For a feedback level $\gamma=0.5 \mathrm{GHz}$ [see Figs. 1(d) and 1(e)] we

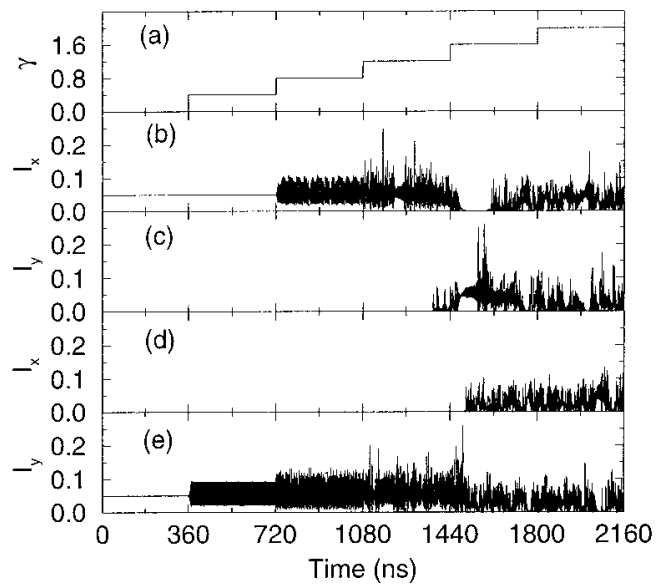

FIG. 3. Effect of increasing the feedback level. The parameters are $\gamma_{a}=0.1 \mathrm{~ns}^{-1}, \gamma_{p}=6 \mathrm{~ns}^{-1}, \gamma_{s}=50 \mathrm{~ns}^{-1}$, and $\mu=1.05$. (a) Timedependent feedback level (in GHz). (b),(c) Intensities (in arbitrary units) of the $x$ - and $y$-polarized components when the initial conditions are chosen in the $x$-polarized state of the solitary laser, respectively. (d),(e) Intensities (in arbitrary units) of the $x$ - and $y$-polarized components, respectively, when the initial conditions are chosen in the $y$-polarized state of the solitary laser. 

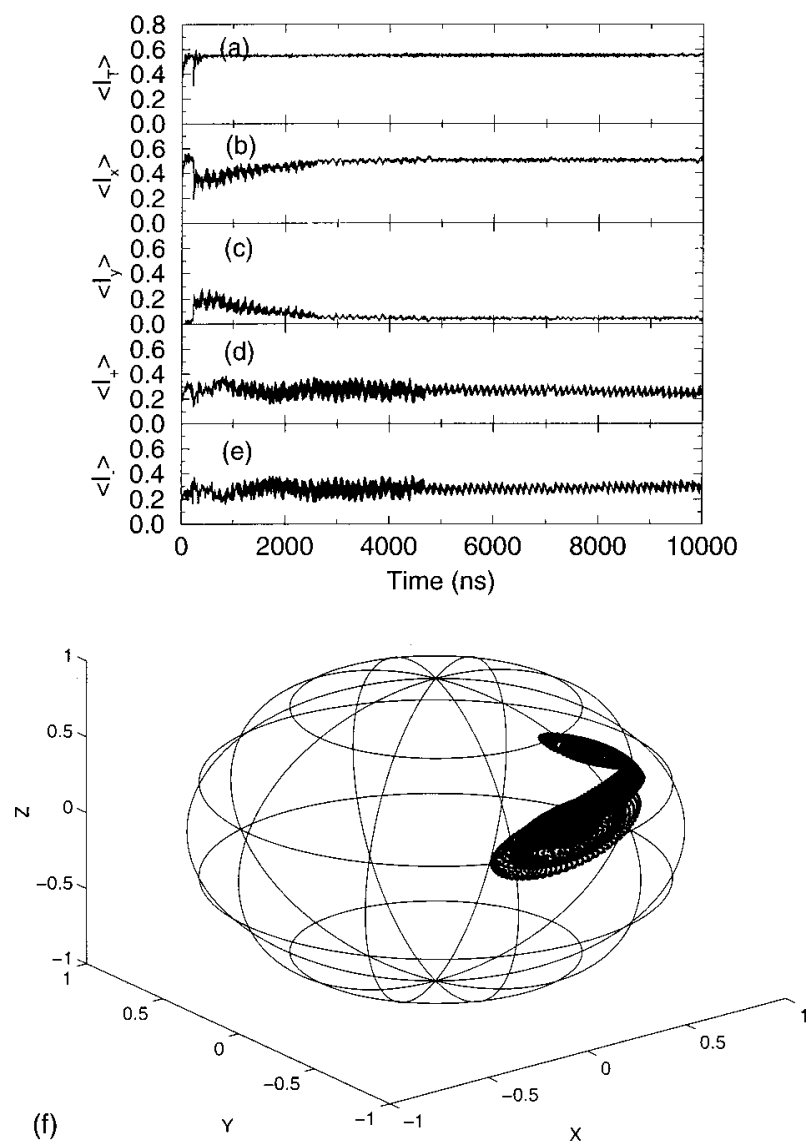

FIG. 4. Total intensity (a), intensities of $x$ - (b), and $y$ - (c) polarized components, and intensities of the right (d) and left (e) circularly polarized components, averaged over 1 ns. (f) Representation on the Poincare sphere of the instantaneous intensity. The parameters are $\gamma_{a}=-0.1 \mathrm{~ns}^{-1}, \gamma_{p}=6 \mathrm{~ns}^{-1}, \gamma_{s}=50 \mathrm{~ns}^{-1}, \mu=1.3$, and $\gamma$ $=50 \mathrm{GHz}$. The $x$ - and $y$-polarized states are stable for the solitary laser. The initial conditions are chosen in the $x$-polarized state of the solitary laser.

also observe a state of mixed polarization before the polarization switching occurs. With weak feedback there is a periodic modulation of the intensity of the linearly polarized states, since weak feedback excites sustained intensity modulation as it does in conventional semiconductor lasers.

Figure 2 shows a switching from the $y$-polarized state to the $x$-polarized state [here again Figs. 2(b) and 2(c) are for no feedback, and Figs. 2(d) and 2(e) are for feedback]. When the feedback level is $\gamma=0.5 \mathrm{GHz}$, the polarization switch occurs at a lower value of the injection current than for the laser without feedback.

Figure 3 shows the effect of increasing the feedback level while keeping the value of the injection current constant in a region of parameters in which the laser without feedback is bistable. For Figs. 3(a) and 3(b), the initial conditions are chosen in the $x$-polarized state, and for Figs. 3(c) and 3(d) in the $y$-polarized state. With low feedback the intensity maintains its polarization state but fluctuates chaotically. If the feedback level is increased above a certain value, the output is not linearly polarized but has two orthogonal linearly polarized components that fluctuate very rapidly (this occurs even in parameter regions where the solitary laser is monostable). The description of the polarization fluctuations
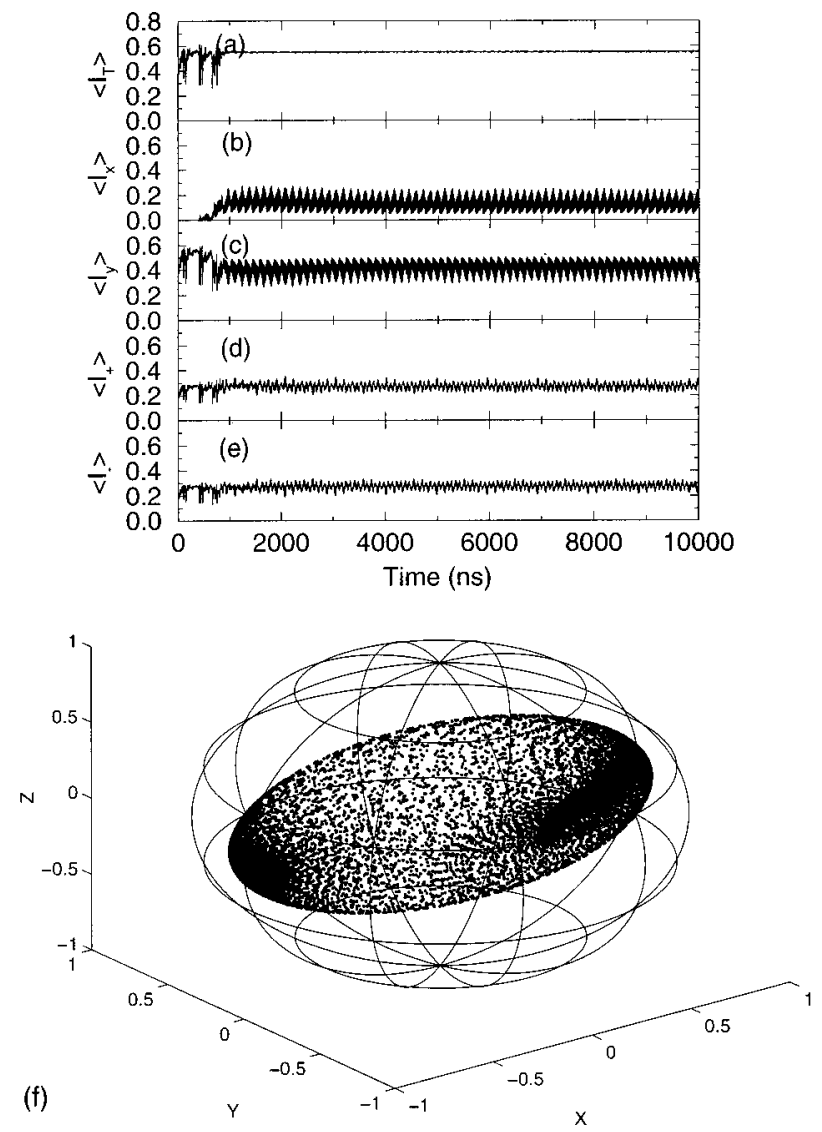

FIG. 5. As in Fig. 4, but the initial conditions are chosen in the $y$-polarized state of the solitary laser.

in this case in terms of competing states is difficult, since the amplitude variables used in the rate equations are the result of an arbitrary selection of basis states for the electric field. Even the basis states that diagonalize the linear terms in the rate equations will not provide an ideal set for the dynamics because of the nonlinear interactions. Depending on the value of the spin-flip relaxation rate, it can be more convenient to select linearly (for large values of $\gamma_{s}$ ) or circularly (for low values of $\gamma_{s}$ ) polarized basis states. For intermediate values of $\gamma_{s}$, the best basis set for a description of the polarization fluctuations, if it exists, probably consists of elliptically polarized states.

The elliptically polarized steady states (which appear above the value of the injection current at which the linearly polarized states of the solitary laser lose stability [9]) are stable in very narrow parameter regions and are easily destabilized by optical feedback.

For moderate feedback we usually find solutions with time-dependent polarization. Averaging the intensity over 1 ns (to simulate the finite bandwidth of the detectors used in most experiments), the total and modal intensities of the linearly polarized components and of the circularly polarized components fluctuate chaotically.

\section{B. Dynamics for moderately strong optical feedback}

For low injection currents $(\mu<1.4-1.5)$ and for moderately strong feedback levels (roughly $\gamma>30 \mathrm{GHz}$ ), different dynamic regimes are found. Each regime exists for a range of values of $\gamma_{s}$. For fast spin relaxation (roughly $\gamma_{s}$ 


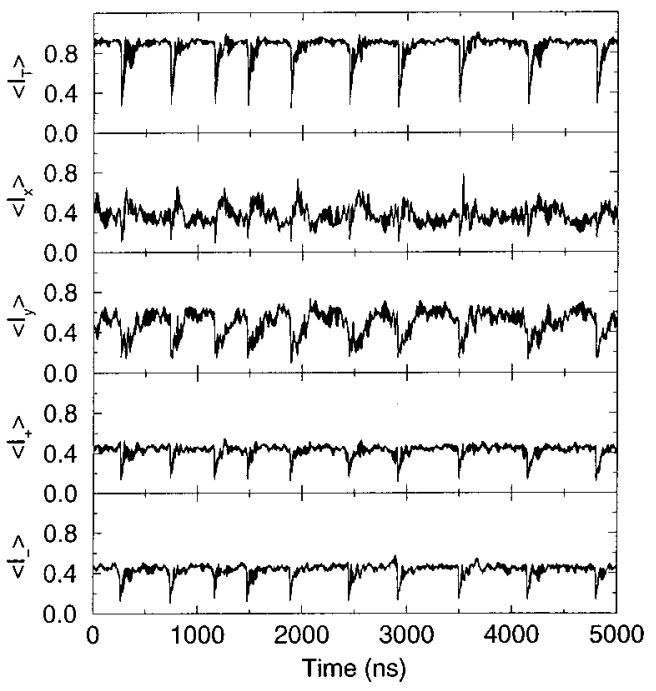

FIG. 6. Time-averaged total intensity, intensities of $x$ - and $y$-polarized components, and intensities of the left and right circularly polarized components. The parameters are $\gamma_{a}=-0.1 \mathrm{~ns}^{-1}$, $\gamma_{p}=4 \mathrm{~ns}^{-1}, \gamma_{s}=10 \mathrm{~ns}^{-1}, \mu=1.3$, and $\gamma=100 \mathrm{GHz}$.

$>40 \mathrm{~ns}^{-1}$ ) the time-averaged total intensity is either constant or approximately constant, depending on the stability of the linearly polarized states of the solitary laser. In this parameter region, either one or both of the two linearly polarized states are stable. When the laser without feedback is monostable, the laser with feedback tends to operate on one of the stable linearly polarized stationary solutions introduced by the feedback [they are the solutions of either Eqs. (11) and (12) or Eqs. (13) and (14) with $\omega_{s}^{i} \ll \omega_{o}$, and the behavior is analogous to that of conventional semiconductor lasers [54]].

In the parameter regions where the laser without feedback is bistable, the dynamics of the laser with feedback depends on the initial conditions. The laser might operate in either one of the stable linearly polarized stationary solutions, or in a state with mixed time-dependent polarization. In the latter case the intensities of the linearly and circularly polarized components fluctuate very rapidly and there is antiphase dynamics [55] of the time-averaged modal intensities that makes the total time-averaged intensity nearly constant.

To characterize the polarization state of the light, we use the Poincaré-sphere plot, where for a given pair of field amplitude components $E_{x}(t) E_{y}(t)$ we assign a point on the Poincaré sphere whose coordinates are

$$
\begin{gathered}
x(t)=\frac{\left|E_{x}(t)\right|^{2}-\left|E_{y}(t)\right|^{2}}{\left|E_{x}(t)\right|^{2}+\left|E_{y}(t)\right|^{2}}, \quad y(t)=\frac{2 \operatorname{Re}\left[E_{x}(t) E_{y}^{*}(t)\right]}{\left|E_{x}(t)\right|^{2}+\left|E_{y}(t)\right|^{2}}, \\
z(t)=\frac{2 \operatorname{Im}\left[E_{x}(t) E_{y}^{*}(t)\right]}{\left|E_{x}(t)\right|^{2}+\left|E_{y}(t)\right|^{2}} .
\end{gathered}
$$

The points in the Poincaré sphere of unit radius are in one-to-one correspondence with the different polarization states of the laser beam (the south pole represents leftcircular polarization, the north pole represents right-circular polarization, the positive $x$ axis represents $x$ polarization, while the negative $x$ axis represents $y$ polarization).

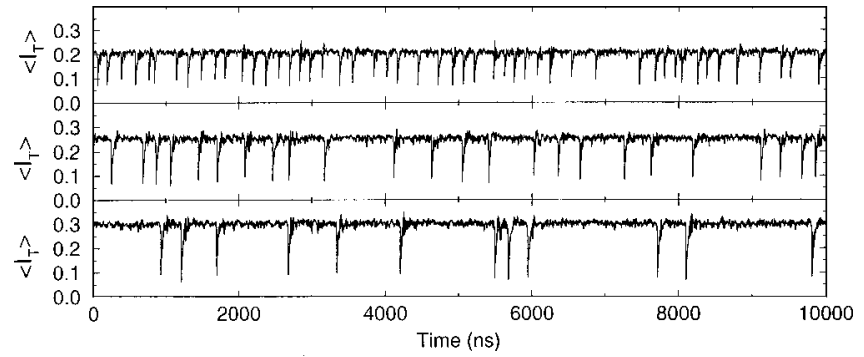

FIG. 7. Time dependence of the total time-averaged intensity for parameters $\gamma_{a}=0.5 \mathrm{~ns}^{-1}, \quad \gamma_{p}=8 \mathrm{~ns}^{-1}, \quad \gamma_{s}=10 \mathrm{~ns}^{-1}, \quad$ and $\mu=1.1$. (a) $\gamma=30 \mathrm{GHz}$, (b) $\gamma=40 \mathrm{GHz}$, and (c) $\gamma=50 \mathrm{GHz}$.

As an example, in Figs. 4 and 5 we show the timeaveraged total and modal intensities (the intensities were averaged over $1 \mathrm{~ns}$ ), taking initial conditions in the $x$ - and $y$-polarized states of the solitary laser, respectively, and the polarization state representation of the instantaneous signal on the Poincaré sphere. On the Poincaré sphere, in the first case there seems to be a competition between two elliptically polarized states, while in the second case, there seems to be a competition between an elliptically polarized state and the $y$-polarized state.

For slow spin relaxation (roughly $\gamma_{s}<40 \mathrm{~ns}^{-1}$ ) the total time-averaged intensity is not constant but shows abrupt dropouts (Fig. 6). When a dropout occurs, the time-averaged intensities $I_{x}, I_{y}, I_{+}$, and $I_{-}$vary in such a way that the total time-averaged intensity markedly decreases. In between the dropouts, there are anticorrelated variations of the timeaveraged modal intensities that keep the total time-averaged intensity nearly constant.

The dropouts in the total intensity present the same striking features as those occurring in conventional semiconductor lasers. They occur suddenly, and are followed by stepwise recoveries (the width of the steps in the recovery phase is the external cavity round-trip time). The dropouts become less frequent and eventually disappear for increasing feedback levels (Fig. 7) or decreasing injection current.

Underlying the dropouts, there is a fast pulsing behavior of the total and modal intensities. A dropout is accompanied by a brusque increase of the phase delays of the linearly and circularly polarized components $\Delta \phi_{x, y}=\phi_{x, y}(t)-\phi_{x, y}(t$ $-\tau), \Delta \phi_{ \pm}=\phi_{ \pm}(t)-\phi_{ \pm}(t-\tau)$, and then they slowly decrease back again during the build-up phase (Fig. 8). There is an antiphase dynamics of the time-averaged intensities of the linearly and circularly polarized components before the dropout occurs, which reappears during the build-up phase.

While the dynamics for weak feedback described in Sec. III A is rather sensitive to the value of the feedback phase $\omega_{o} \tau$ (since variations of $\omega_{o} \tau$ shift the external field from constructive to destructive interference), the dynamics of the time-averaged intensity dropouts that occur for moderate feedback is independent of the value of $\omega_{o} \tau$.

The role of $\gamma_{s}$ in causing (or allowing) the dropouts requires further investigation, but the appearance of dropouts seems to be a consequence of a reduced stability of the linearly polarized states of the solitary VCSEL. Although in the absence of anisotropies the linearly polarized states of the solitary laser are stable for all values of $\gamma_{s}$ (they become marginally stable when $\gamma_{s} \rightarrow \infty$ [6]), in the presence of anisotropies, if $\gamma_{s}$ is below a certain value the circularly 

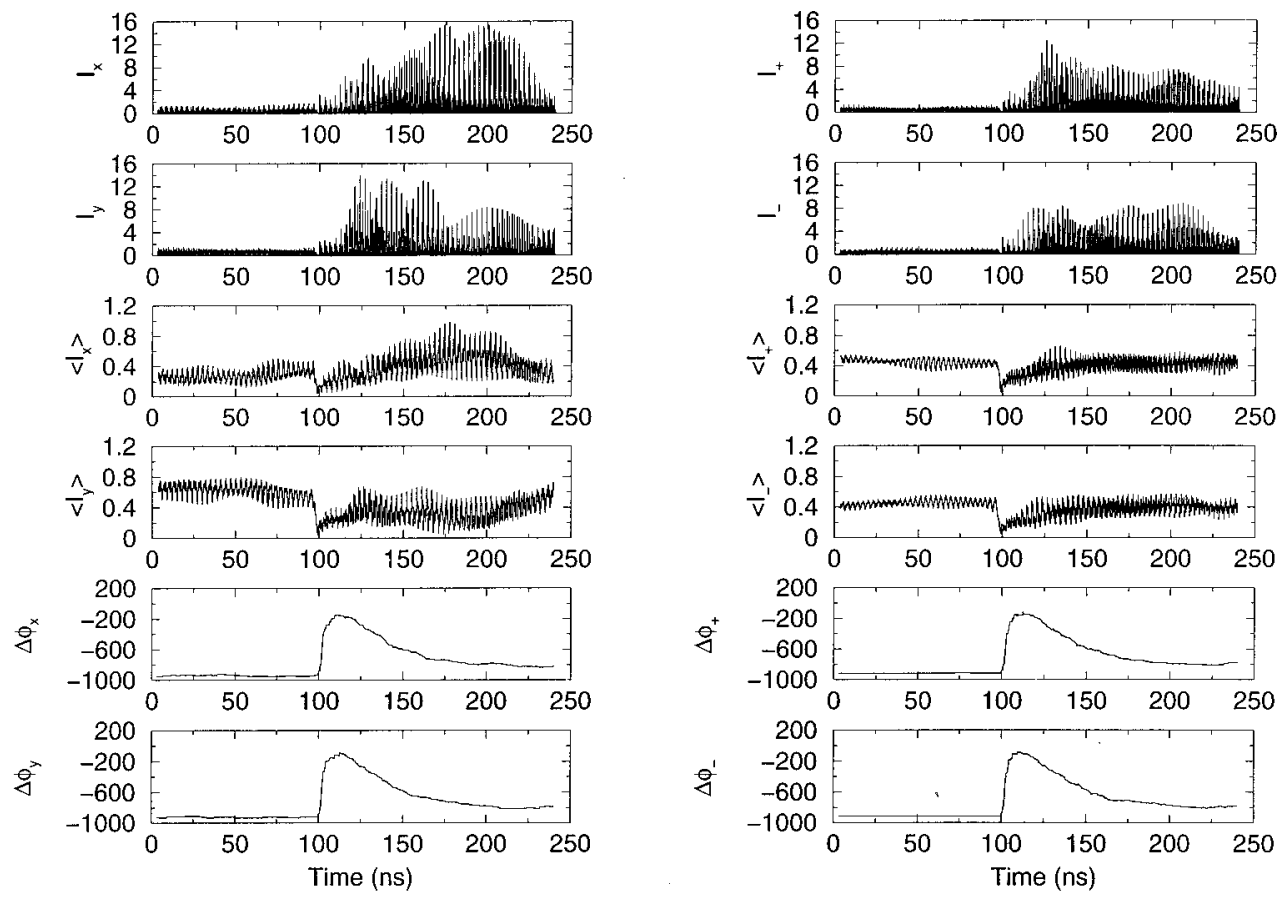

FIG. 8. Time evolution of the modal intensities and of the phase delays during the dropouts. The parameters are as in Fig. 6.

polarized states of the solitary laser fail to phase lock to form stable linearly (or elliptically) polarized states.

In the limiting case of $\gamma_{s}=\gamma_{N}=1 \mathrm{~ns}^{-1}$ [in which each modal amplitude $E_{ \pm}$is coupled to a different population variable $N_{ \pm}$, respectively, but the amplitudes are linearly mixed by anisotropies as indicated in Eqs. (8) and (9)], the $I_{+}$and $I_{-}$time-averaged intensities show dropouts that are generally not simultaneous (Figs. 9 and 10). This causes the total time-averaged intensity dropouts to be less pronounced (the total time-averaged intensity drops to approximately half of its value) and more irregular.

On the contrary, in the limiting case of very fast spin relaxation $\left[\gamma_{s} \rightarrow \infty\right.$, which corresponds to integrating Eqs. (5)-(7), i.e., two-mode Lang-Kobayashi equations], in the presence of birefringence, gain anisotropy, and moderately strong feedback usually one of the two linearly polarized modes wins, and the laser output is constant (antiphase dynamics of the time-averaged modal intensities is observed in the transient evolution to the stable steady state). This occurs for feedback levels for which, when Eqs. (1)-(4) are simulated with the same parameters but with low values of $\gamma_{s}$, dropouts are observed (the dropouts tend to disappear as $\gamma_{s}$ increases).

It is worth noticing that noisy dropouts are observed in Eqs. (5)-(7), but with lower feedback levels, and they tend to disappear as the feedback increases, since usually one of the two linearly polarized modes wins. However, when selfand cross-gain saturation terms are included in the rate equations (5)-(7), power dropouts occur for moderately strong feedback, if the coupling is strong enough. Figure 11 shows the results of simulating the rate equations,

$$
\begin{aligned}
\dot{E}_{x}= & k(1+i \alpha)\left(N f_{x}-1\right) E_{x}-\left(\gamma_{a}+i \gamma_{p}\right) E_{x} \\
& +\gamma E_{x}(t-\tau) e^{-i \omega_{o} \tau}
\end{aligned}
$$

$$
\begin{aligned}
\dot{E}_{y}= & k(1+i \alpha)\left(N f_{y}-1\right) E_{y}+\left(\gamma_{a}+i \gamma_{p}\right) E_{y} \\
& +\gamma E_{y}(t-\tau) e^{-i \omega_{o} \tau}, \\
\dot{N}= & -\gamma_{N}\left[N-\mu+N f_{x}\left|E_{x}\right|^{2}+N f_{y}\left|E_{y}\right|^{2}\right],
\end{aligned}
$$

with $\quad f_{x}=1-\varepsilon_{x x}\left|E_{x}\right|^{2}-\varepsilon_{x y}\left|E_{y}\right|^{2}, \quad f_{y}=1-\varepsilon_{y y}\left|E_{y}\right|^{2}$ $-\varepsilon_{y x}\left|E_{x}\right|^{2}$. The parameters are $\gamma_{a}=-0.1 \mathrm{~ns}^{-1}, \quad \gamma_{p}$ $=4 \mathrm{~ns}^{-1}, \gamma_{s}=50 \mathrm{~ns}^{-1}, \mu=1.3$, and $\gamma=100 \mathrm{GHz}$ (the same as in Fig. 6), and $\varepsilon_{x x}=\varepsilon_{x y}=\varepsilon_{y x}=\varepsilon_{y y}=\varepsilon=0.1$. The modes drop out simultaneously, as it occurs in the SFM model with intermediate values of $\gamma_{s}$ (compare with Fig. 6). However, the mechanism that triggers the dropouts seems to be different. While in the SFM model it is related with a reduced stability of the linearly polarized states of the solitary laser

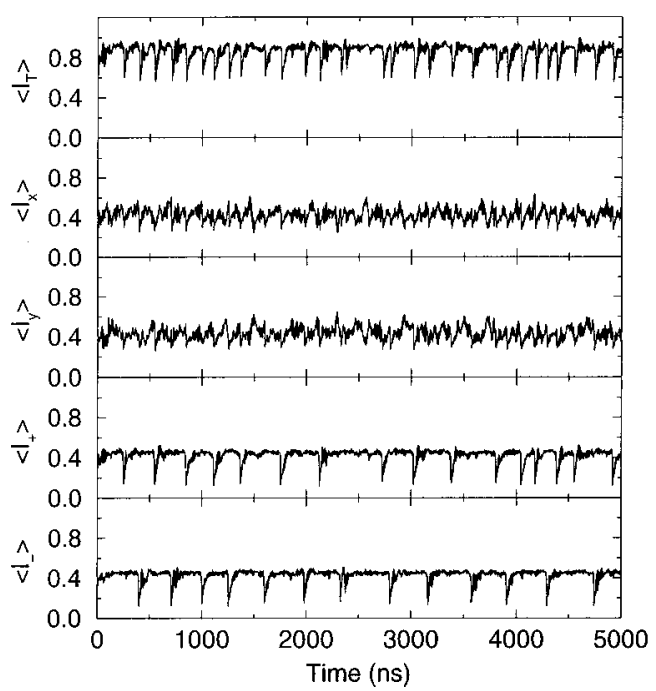

FIG. 9. As in Fig. 6 but with $\gamma_{s}=1 \mathrm{~ns}^{-1}$. 

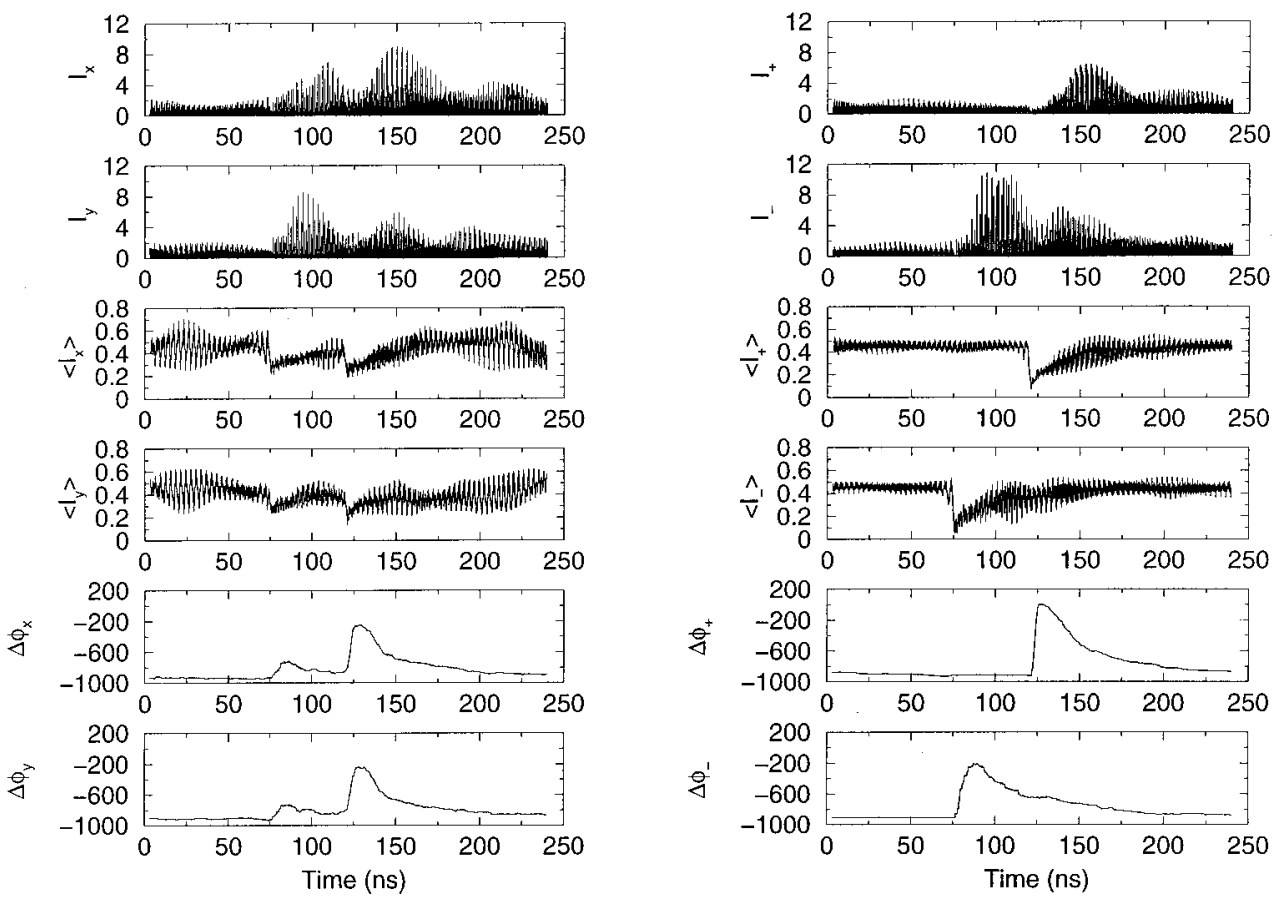

FIG. 10. As in Fig. 8 but with $\gamma_{s}=1 \mathrm{~ns}^{-1}$.

for low values of $\gamma_{s}$, in the model of Eqs. (22)-(24) it is the coupling introduced by the nonlinear gain which breaks the antiphase dynamics and induces dropouts. For the parameters of Fig. 11 and $\varepsilon=0$ the $x$-polarized state is the stable solution, but if we gradually increase the value of $\varepsilon$ we observe antiphase dynamics of the time-averaged modal intensities, until a certain critical value of $\varepsilon$ above which dropouts appear. The dropouts become more frequent as $\varepsilon$ increases, but the time-averaged intensity drops always to the same value, independent of $\varepsilon$.

Although the mean values of the modal intensities are approximately the same, the fluctuations of the instantaneous modal intensities after a dropout are much larger in the SFM model than in the model of Eqs. (22)-(24). It was shown in

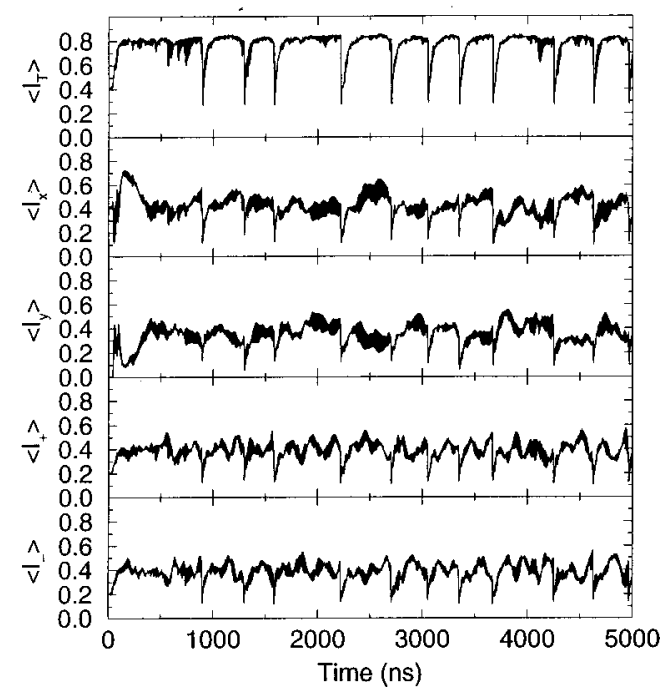

FIG. 11. As in Fig. 6, but simulating the rate equations (21)(24) with $\gamma_{a}=0.1 \mathrm{~ns}^{-1}, \gamma_{p}=4 \mathrm{~ns}^{-1}, \mu=1.3, \quad \gamma=100 \mathrm{GHz}$, and $\varepsilon_{x x}=\varepsilon_{x y}=\varepsilon_{y x}=\varepsilon_{y y}=0.1$.
[43] that the statistics of the intensity fluctuations in the LFF regime of conventional semiconductor lasers differs from the predictions of the Lang-Kobayashi model. It will be interesting to compare our results with experimental results of the statistics of intensity fluctuations in the LFF regime of VCSEL's (when they become available), in order to determine which model (if any) reproduces the experimental finding.

For comparison, in Fig. 12 we show the results of simulating the scalar Lang-Kobayashi equations, where the dynamics is governed by a single complex electric field amplitude and a single population variable $\left(n=0, E_{x}=E_{y}=E / 2\right.$, and $\gamma_{a}=\gamma_{p}=0$ in our equations). In this case the time-
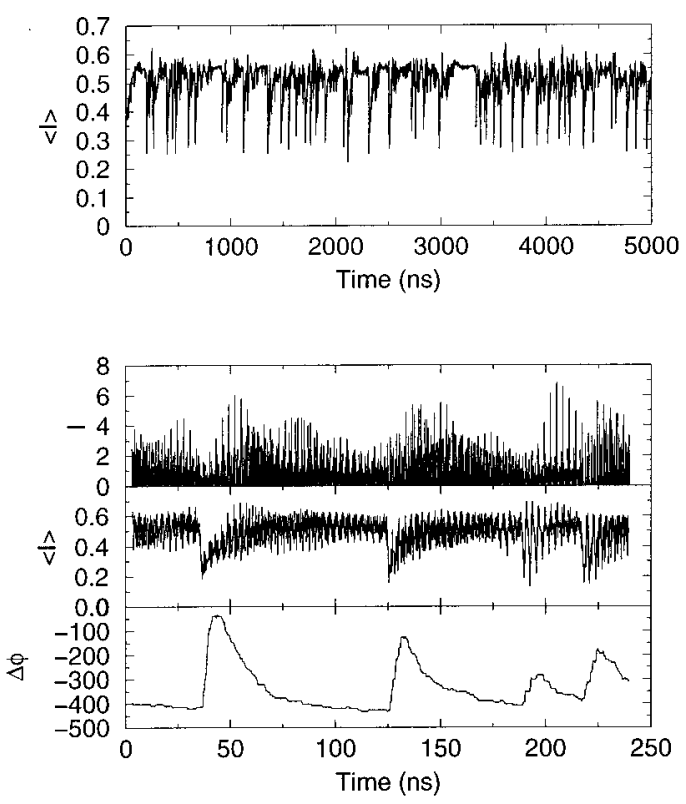

FIG. 12. Simulation of the single-mode model. The parameters are $\gamma_{a}=\gamma_{p}=0 \mathrm{~ns}^{-1}, \mu=1.3$, and $\gamma=50 \mathrm{GHz}$. 


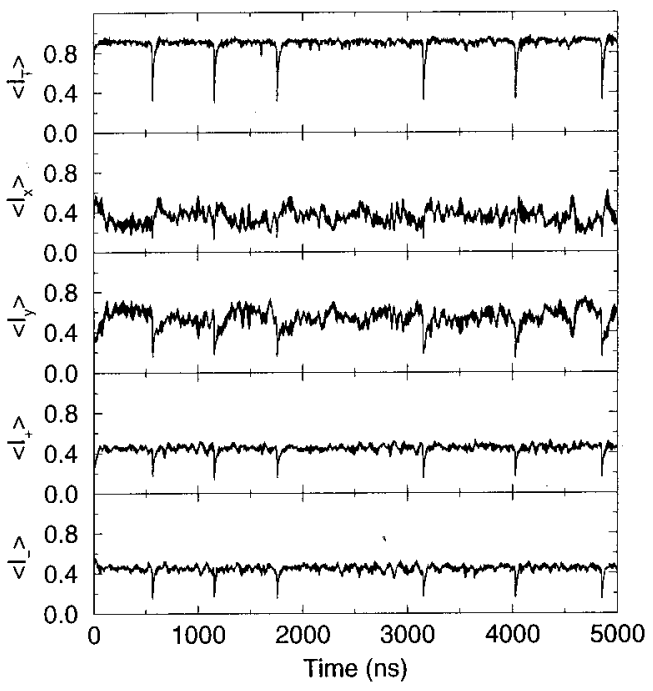

FIG. 13. Effect of noise in the time-averaged total and modal intensities. The parameters are as in Fig. 6 and the noise level is 100 $\mathrm{ns}^{-1}$.

averaged intensity dropouts are much more irregular than those that occur when there is antiphase competition of the two components of a vectorial electric field, and for increasing feedback the dropouts become a feature of the transient to a stationary solution.

\section{Effect of spontaneous emission noise}

To investigate which features of the dynamics are essentially due to the mode competition and which might be noise-induced, we increased the Langevin noise terms of the field rate equations (to strengths comparable to the feedback) and studied how this noise variation modified the dynamics.

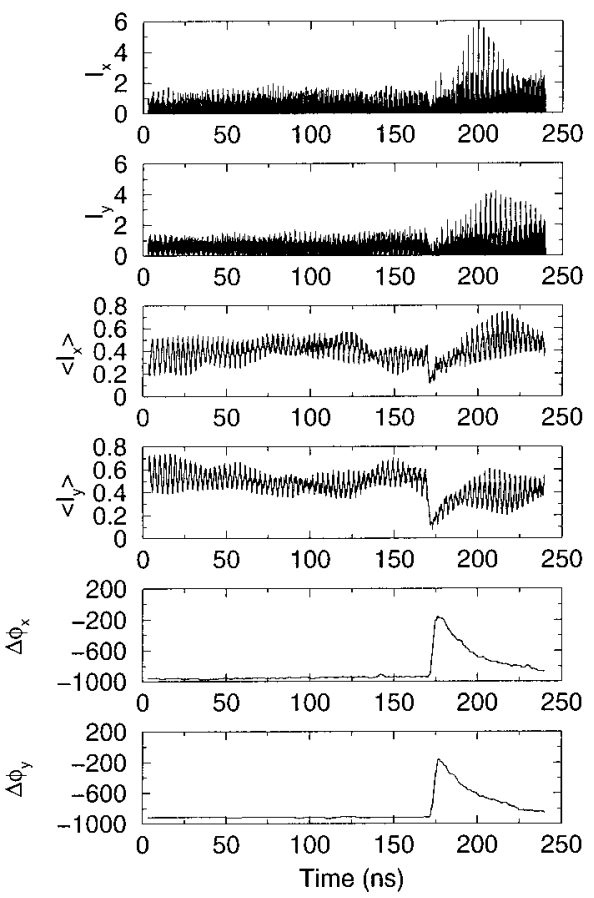

In the four cases studied above $\left(\gamma_{s}=1 \mathrm{~ns}^{-1}, \quad \gamma_{s}\right.$ $=10 \mathrm{~ns}^{-1}, \gamma_{s}=\infty$, and the Lang-Kobayashi equations), the effect of noise is to make the dropouts less frequent (for the Lang-Kobayashi model this was discussed by Hohl et al. [37]). As an example, we show in Figs. 13 and 14 the timeaveraged modal intensities, the instantaneous modal intensities, and the phase delay variations during the dropouts, for the same parameters as Figs. 6 and 8 and a noise level of $\beta=100 \mathrm{~ns}^{-1}$.

In the SFM model noise does not modify the mean values of the time-averaged intensities, but makes the fluctuations of the instantaneous intensities after a dropout considerably smaller (compare the scales of the vertical axis of Figs. 8 and 14). Also, noise often causes more "abrupt" phase delay shifts during the dropouts. There are parameter regions where in the absence of noise no dropouts occur but antiphase dynamics of the time-averaged modal intensities exist, and in which sufficiently high noise levels break the antiphase dynamics and induce intensity dropouts (the total time-averaged intensity drops to a value that is independent of the noise level, but the frequency of the dropouts increases with the noise level).

\section{SUMMARY AND CONCLUSIONS}

We have studied in detail the dynamics of a VCSEL operating near threshold and with isotropic optical feedback. Weak feedback levels induce the same instabilities as in conventional polarization-stabilized semiconductor lasers: the relaxation oscillations become undamped and multiple steady-state solutions are created (a series of external cavity modes for each linearly polarized state of the solitary laser). Since the optical feedback that we consider is isotropic, weak feedback levels usually do not modify the polarization state of the laser, which is the same as the solitary laser.
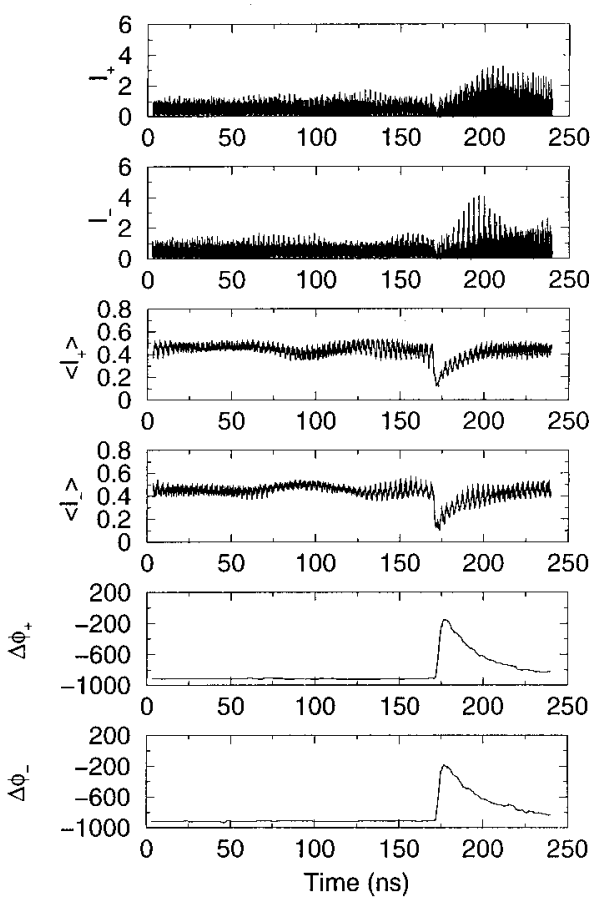

FIG. 14. Effect of noise in the time evolution of the modal intensities and of the phase delays during the dropouts. The parameters are as in Fig. 13. 
However, for moderately strong feedback levels the vectorial degree of freedom of the electric field in VCSEL's adds new interesting features to the dynamics, which are sensitive to the value of the spin-flip relaxation rate. For low and intermediate values of $\gamma_{s}$ the total time-averaged intensity shows randomly occurring sudden dropouts that have the same characteristics as those occurring in conventional laser diodes. The dropouts tend to disappear for increasing $\gamma_{s}$, and this seems to be related with the fact that in the presence of birefringence and gain anisotropy, one of the two linearly polarized states of the solitary laser usually becomes stable as $\gamma_{s}$ increases. If only one of the linearly polarized states is stable, the laser tends to operate on one of its stable stationary solutions; if both states are stable, the laser might operate in one of these stable stationary solutions, or there might be antiphase dynamics of the time-averaged modal intensities that makes the total time-averaged intensity nearly constant (underlying the antiphase dynamics, there is a fast pulsing behavior of the instantaneous modal intensities).

An explanation for the dropouts in conventional singlemode semiconductor lasers, originally proposed by Sano [36], relies on a chaotic itinerancy among destabilized exter- nal cavity modes. In this interpretation a dropout occurs because the trajectory (in its attempt to reach a stable stationary solution) collides with an antimode (i.e., a saddle stationary solution). Our results indicate that in VCSEL's, in which the fundamental transverse mode has two orthogonal linearly polarized states, the collision with an antimode is not the only mechanism that might induce a dropout. We found situations in which if the intensity of one linearly polarized mode undergoes a dropout, the intensity of the orthogonal mode follows it and drops out almost simultaneously. This occurs when the two linearly polarized modes interact with each other through a single carrier density while the nonlinear gain is important, and when the two modes interact with each other through two carriers densities, while the spin relaxation rate takes intermediate values. We have also studied the effect of noise, showing that noise does not modify the essential features of the dynamics.

\section{ACKNOWLEDGMENTS}

This work was supported in part by the Comisión Sectorial de Investigación Cientifica (CSIC) and the PEDECIBA.
[1] J. Martin-Regalado, J. L. A. Chilla, J. J. Rocca, and P. Brusenbach, Appl. Phys. Lett. 70, 3350 (1997).

[2] K. D. Choquette, D. A. Richtie, and R. E. Leibenguth, Appl. Phys. Lett. 64, 2062 (1994).

[3] A. K. Jansen van Doorn, M. P. van Exter, M. Travagnin, and J. P. Woerdman, Opt. Commun. 133, 252 (1997).

[4] C. J. Chang-Hasnain, J. P. Harbison, G. Hasnain, A. C. Von Lehmen, L. T. Florez, and N. G. Stoffel, IEEE J. Quantum Electron. 27, 1402 (1991).

[5] K. D. Choquette, R. P. Schneider, Jr., K. L. Lear, and R. E. Leibenguth, IEEE J. Sel. Top. Quantum Electron. 1, 661 (1995).

[6] M. San Miguel, Q. Feng, and J. V. Moloney, Phys. Rev. A 52, 1728 (1995).

[7] N. B. Abraham, E. Arimondo, and M. San Miguel, Opt. Commun. 117, 344 (1995); 121, 168(E) (1995).

[8] J. Martin-Regalado, M. San Miguel, N. B. Abraham, and F. Prati, Opt. Lett. 21, 351 (1996).

[9] J. Martin-Regalado, F. Prati, M. San Miguel, and N. B. Abraham, IEEE J. Quantum Electron. 33, 765 (1997).

[10] H. van der Lem and D. Lenstra, Opt. Lett. 22, 1698 (1997).

[11] M. P. van Exter, R. F. M. Hendriks, and J. P. Woerdman, Phys. Rev. A 57, 2080 (1998).

[12] M. Travagnin, M. P. van Exter, A. K. Jansen van Doorn, and J. P. Woerdman, Phys. Rev. A 54, 1647 (1996).

[13] A. Gahl, S. Balle, and M. San Miguel, Quantum Semiclassic. Opt. 10, L1 (1998).

[14] C. Serrat, N. B. Abraham, M. San Miguel, R. Vilaseca, and J. Martin-Regalado, Phys. Rev. A 53, R3731 (1996).

[15] T. Mukaihara, N. Ohnoki, Y. Hayashi, N. Hatori, F. Koyama, and K. Iga, IEEE Photonics Technol. Lett. 7, 1113 (1995).

[16] D. Sun, E. Towe, P. H. Ostdiek, J. W. Grantham, and G. J. Vansuch, IEEE J. Sel. Top. Quantum Electron. 1, 674 (1995).

[17] T. Mukaihara, N. Ohnoki, Y. Hayashi, N. Hatori, F. Koyama, and K. Iga, IEEE J. Sel. Top. Quantum Electron. 1, 667 (1995).

[18] K. D. Choquette and R. E. Leibenguth, IEEE Photonics Technol. Lett. 6, 40 (1994).

[19] Z. G. Pan, S. Jiang, M. Dagenais, R. A. Morgan, K. Kojima, M. T. Asom, R. E. Leibenguth, G. D. Guth, and M. W. Focht, Appl. Phys. Lett. 63, 2999 (1993).

[20] K. D. Choquette, K. L. Lear, R. E. Leibenguth, and M. T. Asom, Appl. Phys. Lett. 64, 2767 (1994).

[21] F. Robert, P. Besnard, M.-L. Charès, and G. M. Stéphan, Opt. Quantum Electron. 27, 805 (1995).

[22] S. Jiang, Z. Pan, M. Dagenais, R. A. Morgan, and K. Kojima, Appl. Phys. Lett. 63, 3545 (1993).

[23] D. V. Kuksenkov and H. Temkin, IEEE J. Sel. Top. Quantum Electron. 3, 390 (1997).

[24] L. Hua, A. Hohl, A. Gavrielides, H. Hong, and K. D. Choquette, Appl. Phys. Lett. 72, 2355 (1998).

[25] G. C. Wilson, M. A. Hadley, J. S. Smith, and K. Y. Lau, Appl. Phys. Lett. 63, 3265 (1993).

[26] N. Yokouchi, T. Miyamoto, T. Uchida, Y. Inaba, F. Koyama, and K. Iga, IEEE Photonics Technol. Lett. 4, 701 (1992).

[27] K. Petermann, IEEE J. Sel. Top. Quantum Electron. 1, 480 (1995).

[28] G. H. M. van Tartwijk and D. Lenstra, Quantum Semiclassic. Opt. 7, 87 (1995).

[29] Y. C. Chung and Y. H. Lee, IEEE Photonics Technol. Lett. 3, 597 (1991).

[30] H. M. Chen, K. Tai, K. F. Huang, Y. H. Kao, and J. D. Wynn, J. Appl. Phys. 73, 16 (1993).

[31] K-P Ho, J. D. Walker, and J. M. Kahn, IEEE Photonics Technol. Lett. 5, 892-895 (1993).

[32] J. W. Bae, H. Temkin, S. E. Swirhun, W. E. Quinn, P. Brusenbach, C. Parsons, M. Kim, and T. Uchida, Appl. Phys. Lett. 63, 1480 (1993). 
[33] S. Jiang, Z. Pan, M. Dagenais, R. A. Morgan, and K. Kojima, IEEE Photonics Technol. Lett. 6, 34 (1994).

[34] U. Fiedler, and K. J. Ebeling, IEEE J. Sel. Top. Quantum Electron. 1, 442 (1995).

[35] R. W. Tkach and A. R. Chraplyvy, J. Lightwave Technol. LT-4, 1655 (1986).

[36] T. Sano, Phys. Rev. A 50, 2719 (1994).

[37] A. Hohl, H. J. C. van der Linden, and R. Roy, Opt. Lett. 20, 2396 (1995).

[38] B. Tromborg, J. Mork, and V. Velichansky, Quantum Semiclassic. Opt. 9, 831 (1997).

[39] I. Fischer, G. H. M. van Tartwijk, A. M. Levine, W. Elsässer, E. Göbel, and D. Lenstra, Phys. Rev. Lett. 76, 220 (1996).

[40] R. Lang and K. Kobayashi, IEEE J. Quantum Electron. QE16, 347 (1980).

[41] G. Huyet, S. Hegarty, M. Giudici, B. de Bruyn, and J. G. McInerney, Europhys. Lett. 40, 619 (1997).

[42] D. W. Sukow, J. R. Gardner, and D. J. Gauthier, Phys. Rev. A 56, R3370 (1997).

[43] G. Huyet, S. Bale, M. Giudici, C. Green, G. Giacomeli, and J. R. Tredicce, Opt. Commun. 149, 341 (1998).
[44] J. Dellunde, A. Valle, and K. A. Shore, J. Opt. Soc. Am. B 13, 2477 (1996).

[45] L. N. Langley and K. A. Shore, IEE Proc.: Optoelectron. 144, 34 (1997).

[46] J. Y. Law and G. P. Agrawal, IEEE J. Sel. Top. Quantum Electron. 3, 353 (1997).

[47] P. Besnard, F. Robert, M.-L. Charès, and G. M. Stéphan, Phys. Rev. A 56, 3191 (1997).

[48] N. A. Loiko, A. V. Naumenko, and N. B. Abraham, Quantum Semiclassic. Opt. 10, 125 (1998).

[49] C. Masoller and N. B. Abraham, Appl. Phys. Lett. (to be published).

[50] M. Born and E. Wolf, Principles of Optics, 6th ed. (Pergamon, Oxford, 1993).

[51] C. H. Henry, IEEE J. Quantum Electron. QE-18, 259 (1982).

[52] M. Oestreich, S. Hallstein, and W. W. Ruhle, IEEE J. Sel. Top. Quantum Electron. 2, 747 (1996).

[53] L. J. Sham, J. Phys.: Condens. Matter 5, A51 (1993).

[54] A. M. Levine, G. H. M. van Tartwijk, D. Lenstra, and T. Erneux, Phys. Rev. A 52, R3436 (1995).

[55] W. Jingyi and P. Mandel, Phys. Rev. A 48, 671 (1993). 